\title{
Nova proposta para ensino/treinamento na detecção de lesões de cárie: insights da implementação do método entre estudantes de graduação
}

\author{
Mariana Minatel Braga*; Tathiane Larissa Lenzi**; Bianca Prado Tibério***; Fernanda Rosche \\ Ferreira****; Fausto Medeiros Mendes*; Kim Rud Ekstrand*****
}

\author{
* Professor Associado, Departamento de Ortodontia e \\ Odontopediatria, Faculdade de Odontologia da Universidade de \\ São Paulo \\ ** Pós-doutoranda, Programa de Pós-Graduação em Ciências \\ Odontológicas, Faculdade de Odontologia da Universidade de São \\ Paulo \\ *** Cirurgiã-dentista, Faculdade de Odontologia da Universidade de \\ São Paulo \\ **** Mestre, Faculdade de Odontologia da Universidade de São Paulo \\ ***** Department of Endodontics and Cariology, University of \\ Copenhagen
}

Recebido em 16/05/2017. Aprovado em 26/11/2017.

\begin{abstract}
RESUMO
O presente estudo propõe um método para o ensino de detecção e avaliação de lesões de cárie, utilizando o Sistema Internacional de Avaliação e Detecção de Cárie (ICDAS) como ferramenta auxiliar. Sessenta e dois estudantes do quarto ano do curso de graduação em Odontologia da Universidade de São Paulo foram submetidos a três atividades de treinamento para uso do ICDAS, aplicadas em diferentes momentos: aula teórica convencional, atividade com projeção de imagens e atividade prática-laboratorial com dentes extraídos. Os estudantes responderam a questionários, antes e após as atividades, para avaliação do conhecimento e da percepção sobre as mesmas. Também foi realizada avaliação prática, com avaliação de dentes extraídos. Após os exercícios laboratoriais, a média de respostas corretas para questões conceituais aumentou significativamente e se manteve até o final da atividade laboratorial. Quem acreditava estar bem preparado no início da atividade teve 3 vezes mais chances de alcançar nota acima de 5 no segundo momento da atividade prática-laboratorial $(\mathrm{OR}=3,1 ; 95 \%$ IC=1,0 - 9,1). Concluiu-se que a atividade prática-laboratorial contribui para o aprendizado de estudantes de graduação na detecção de lesões cárie, inclusive sanando dúvidas conceituais que possam existir após a aula teórica. Todavia, a percepção do estudante pode não ser impactada pela atividade.
\end{abstract}

Descritores: Cárie Dentária. Educação em Odontologia. Odontologia. 


\section{INTRODUÇÃO}

A detecção das lesões de cárie tem sido apontada como um tema obrigatório nas aulas de Cariologia dos cursos de formação de profissionais na área de Odontologia ${ }^{1,2}$. Por outro lado, essa tem sido uma das tarefas mais complexas na Odontologia contemporânea ${ }^{3,4}$, devido à falta de consenso entre diferentes escolas e educadores ${ }^{4}$. Uma tentativa de criar um sistema de escores para utilização mundial, útil nos campos de atuação da epidemiologia, da prática clínica e dos estudos clínicos tem sido realizada nos últimos anos ${ }^{3,5}$.

Nesse intuito, um grupo de pesquisadores criou recentemente o International Caries Detection and Assessment System (ICDAS) $)^{3,5}$, um sistema que considera a detecção das lesões de cárie desde o seu estágio mais inicial de desenvolvimento (lesões não cavitadas) até cavitações extensas ${ }^{3,5}$. Essa abordagem das lesões iniciais tem sido apontada como importante para o estabelecimento de condutas de diagnóstico e terapêutica adequadas e garante melhor prognóstico no tratamento da doença ${ }^{6,7}$. Além disso, uma revisão sistemática recente apontou que o uso de índices melhora a detecção de lesões de cárie realizada pelo exame visual ${ }^{8}$.

O comitê responsável pela criação e pelo treinamento para uso do índice conta com um manual de treinamento e um e-learning, disponíveis em seu respectivo website (www.icdas.org). Estudo prévio demonstrou que o e-learning auxilia no aprendizado do ICDAS pelo estudante de graduação ${ }^{9}$. O e-learning é um programa interativo, mas não permite a comunicação entre estudante e professor/tutor como ocorre com uma atividade prática. Por outro lado, a Disciplina de Graduação de Odontopediatria da Faculdade de Odontologia da USP - São Paulo (FOUSP) tem utilizado, desde 2009, um novo sistema de ensino-aprendizagem para treinar seus estudantes, em atividade pré- clínica, para realizar detecção de lesões de cárie. Essa atividade conta com a participação dos docentes e estudantes de pós-graduação, além dos estudantes de graduação, e compreende a realização de três etapas, sendo uma teórica e outras duas práticas, com imagens e dentes extraídos. Nesse contexto, é extremamente importante que tanto o desempenho como a percepção dos estudantes frente a essas novas atividades sejam avaliadas, a fim de respaldar o uso dessas técnicas no processo de ensinoaprendizagem na graduação.

Dessa forma, este trabalho teve por objetivo descrever os primeiros insights da implementação de um novo método laboratorial para ensino de detecção e avaliação de lesões de cárie utilizando o ICDAS, no desempenho, conhecimento teórico e na percepção dos estudantes de graduação em Odontologia.

\section{METODOLOGIA}

\section{Amostra e descrição da atividade}

O estudo foi aprovado pelo Comitê de Ética institucional (CAAE $n^{\circ}$ 39632614.0.0000.0075). A amostra foi composta pelos questionários e fichas preenchidos pelos estudantes que cursavam o $4^{\circ}$ ano do curso diurno de graduação em Odontologia da Universidade de São Paulo no ano de 2009 ( $n=62)$ e que realizaram as atividades teórico-práticas.

Este trabalho foi baseado na coleta de dados provenientes de uma avaliação intradisciplinar de uma atividade nova, implementada no cronograma do curso de graduação de Odontologia pela Disciplina de Odontopediatria. A atividade foi realizada em agosto de 2009, com a participação dos docentes da disciplina e pós-graduandos a ela vinculados, em dois dias, com intervalo de uma semana entre eles e consistiu das seguintes etapas:

Momento 1 (M1, atividade teórica - $1^{\circ}$ dia): aula teórica convencional (presencial) com 50 
minutos de duração, abordando o tema "Detecção de Lesões de Cárie”. Nessa aula, a detecção de lesões de cárie é contextualizada como parte do processo de diagnóstico da doença cárie. São também abordados os aspectos importantes a serem avaliados nesse processo, tendo em vista seu papel no prognóstico e velocidade de progressão das lesões, bem como as possíveis decisões de tratamento. Por fim, o ICDAS (figura 1) é apresentado como uma ferramenta para auxiliar na detecção das lesões de cárie pelo método de inspeção visual. Nessa apresentação, uma associação é feita entre os escores, evolução da lesão, profundidade histológica e decisão de tratamento.

Momento 2 (M2, laboratorial/atividade com imagens - $2^{\mathbf{0}} \mathbf{d i a}$ ): os estudantes foram divididos em oito grupos compostos por 8 integrantes, em média (figura 2A). Cada um desses grupos foi acompanhado por dois a três estudantes de pósgraduação (tutores). Os estudantes deveriam classificar, de acordo com os escores do ICDAS, os sítios indicados em trinta fotografias clínicas. Após o término da projeção, os tutores discutiram, em seus grupos, os escores atribuídos para cada caso. As imagens pertenciam a um banco de imagens e foram escolhidas com finalidade didática. Um gabarito único foi proposto para nortear as correções e posteriores discussões. Os tutores não apenas tinham conhecimento das respostas, como haviam participado ativamente da mesma atividade, em ocasião prévia, com a finalidade de calibração.

\section{Momento 3 (M3, laboratorial/atividade com} dentes extraídos - $2^{\circ}$ dia): após a discussão de cada grupo com seus tutores, os estudantes avaliaram e classificaram 12 dentes de uma amostra de dentes extraídos preparada exclusivamente para a atividade (figura 2B). Para essa avaliação, os estudantes utilizaram jatos de ar e água (seringa tríplice), iluminação artificial e sonda milimetrada do tipo OMS. Para mimetizar as condições de umidade do ambiente bucal, os estudantes eram orientados a umedecer os dentes previamente às avaliações. Novamente, os tutores interagiam com os estudantes de graduação, corrigindo as respostas dadas e discutindo possíveis divergências que ocorressem dentro de cada grupo.

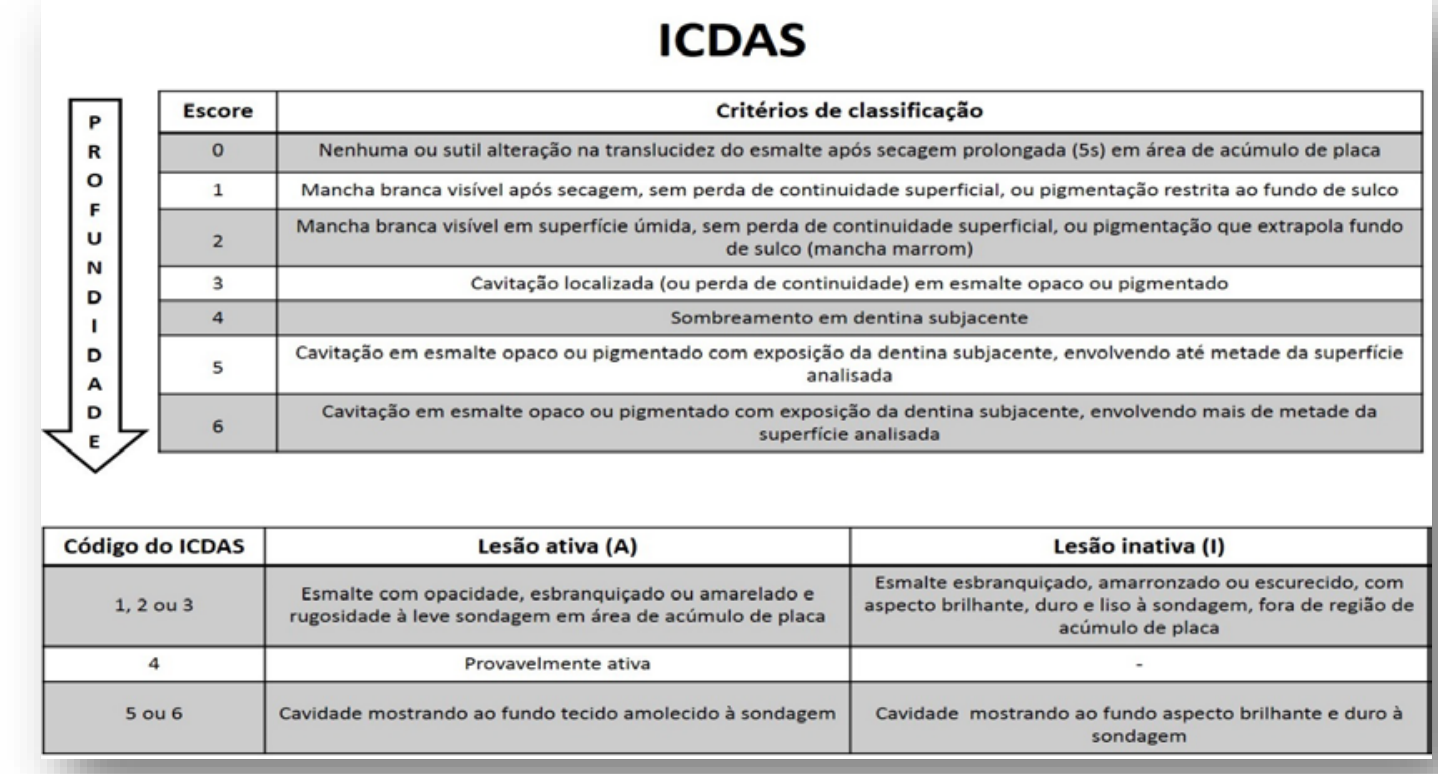

Figura 1. Avaliação da severidade e atividade das lesões de cárie baseada no ICDAS 


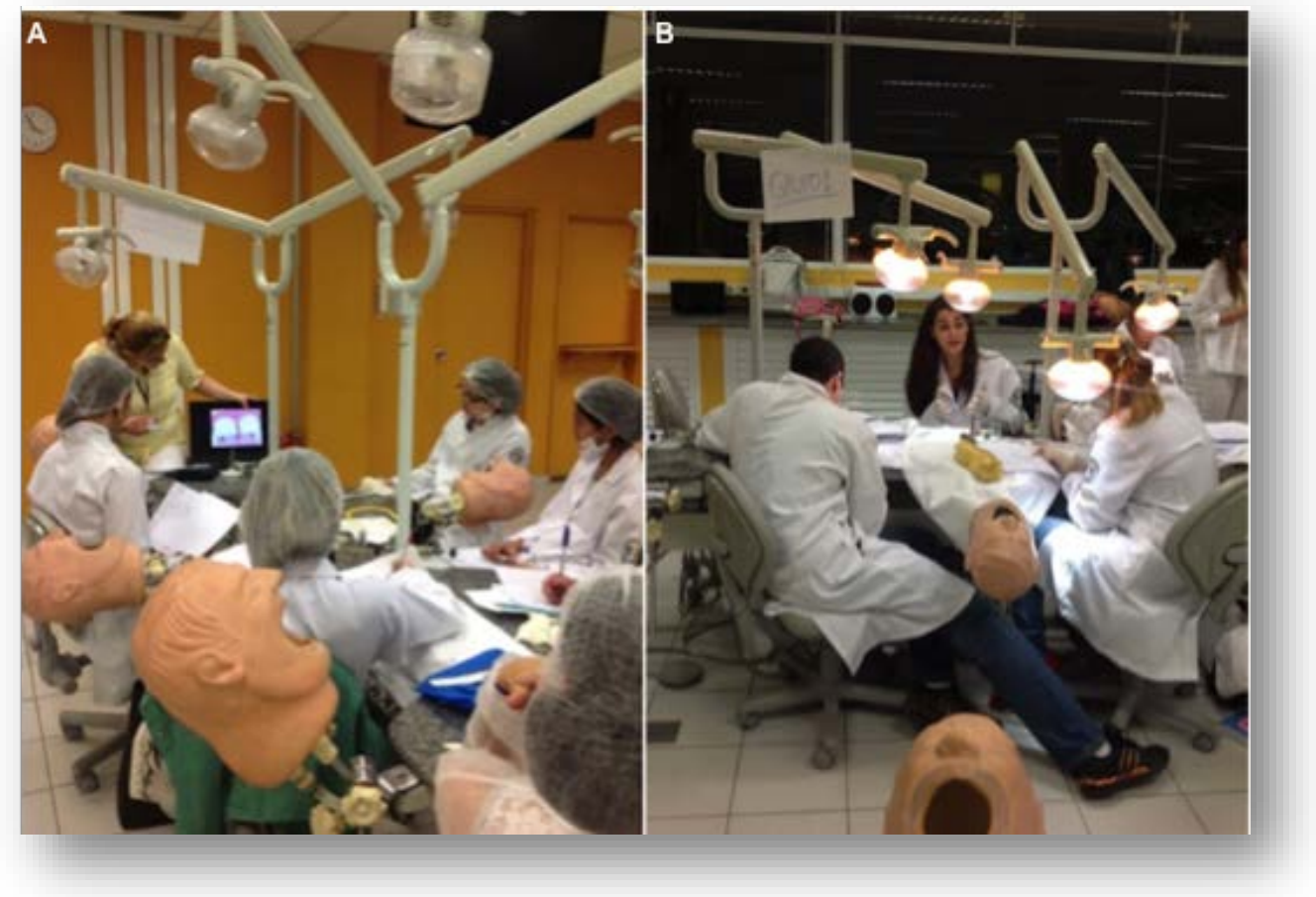

Figura 2. Treinamento prático para detecção de lesões de cárie realizado com fotografias clínicas retiradas de um banco de imagens, com correção e discussão mediada por tutor (A); atividade prática para detecção de lesões de cárie realizada com dentes extraídos, com interação dos estudantes (B)

\section{Avaliação dos resultados}

Para avaliação do conteúdo teórico relativo ao tópico "Detecção de lesões de cárie”, cinco questões de múltipla escolha foram elaboradas pelos docentes e pós-graduandos. Duas questões foram focadas em aspectos conceituais sobre o tema (questões 4 e 5). Outras duas questões abordavam a habilidade em tomar decisões em diferentes circunstâncias descritas sobre detecção de cárie (questões 1 e 3) e, finalmente, uma questão exigia que os estudantes fossem aptos a identificar aspectos importantes relacionados ao ICDAS e reconhecer possíveis consequências do erro de classificação durante o exame clínico (questão 2). Esse questionário foi aplicado logo após a aula teórica, sendo repetido duas vezes, após as atividades práticas com imagens e com dentes extraídos.

Como forma de avaliação prática, optou- se pela utilização de dentes extraídos. Os participantes deveriam avaliar 12 superfícies específicas apontadas. Essa avaliação ocorreu em dois momentos (logo após a aula teórica e após a atividade prática com imagens). Para essas avaliações, um gabarito foi estabelecido por um examinador de referência. O número de acertos em cada uma das atividades foi computado e uma nota de 0 a 10 atribuída para cada uma delas.

Um segundo questionário foi utilizado para avaliação da percepção do estudante frente à atividade aplicada. Trata-se de um questionário de percepção associado à escala "State Trait Personality Inventory"10. Para cada uma das características investigadas, foi usada escala tipo Likert de quatro pontos (Absolutamente não, Pouco, Moderadamente e Muito). Os estudantes responderam a esse questionário nas mesmas ocasiões em que responderam ao questionário 
sobre conhecimento teórico.

Todas as etapas de avaliação foram realizadas no mesmo dia, correspondendo ao $2^{\circ}$ dia de atividade didática.

\section{Análise dos dados}

A relação entre o número de acertos nas diferentes questões teóricas nos três momentos de avaliação foi descrita. As notas obtidas nas avaliações práticas foram descritas por médias e as duas avaliações dos dentes (pré e pós discussão de imagens) foram comparadas pelo teste $t$ de Student.

Análises de regressão linear foram empregadas para verificar a associação entre as notas médias obtidas nas avaliações teórica e prática. Também foi testada, por regressão logística, a associação entre o conhecimento teórico dos estudantes e aspectos de sua percepção sobre a atividade. Para isso foi considerado, como desfecho, acerto superior a 50\% na avaliação teórica.
Para os quesitos relacionados à percepção dos estudantes antes e após a atividade, os dados foram expressos de forma descritiva. Quando necessário, a percepção foi convertida em escala de 0 (absolutamente não) a 3 (muito) para comparação pelo teste de Friedman.

\section{RESULTADOS}

De forma geral, os estudantes apresentaram bom desempenho (70\% de acertos) no teste após a aula teórica (M1). Esse índice foi incrementado em $10 \%$ após a avaliação das imagens, com correção e discussão com os tutores, sem incremento significativo após o treinamento com dentes extraídos (gráfico 1). O treinamento laboratorial incrementou, em especial, o número de acertos nas questões 3 e 5 .

Dos estudantes que cometeram algum erro durante a avaliação teórica, cerca de 50\% acreditavam estar bem preparados para a realização da detecção de lesões de cárie em casos simulados em imagens e dentes extraídos.

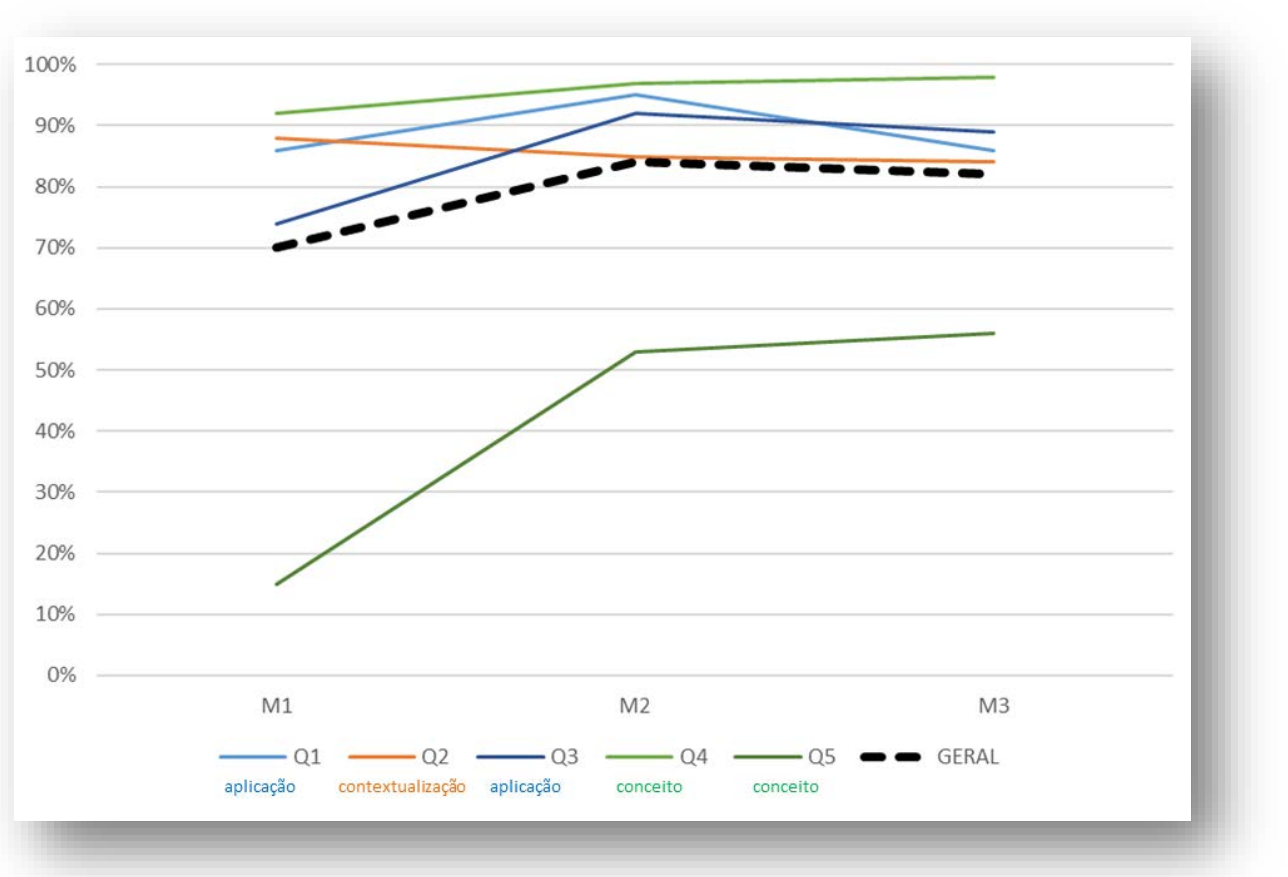

Gráfico 1. Porcentagem de respostas corretas no teste teórico nos três momentos de avaliação. M1: após aula teórica; M2: após avaliação de imagens; e M3: após avaliação de dentes extraídos. Qn: número da questão, associada ao tipo de conhecimento avaliado. 
Os estudantes também mostraram discreta melhora nas notas dos exercícios com dentes extraídos realizados no início $(5,1 \pm 1,4)$ e no final da atividade $(5,3 \pm 1,4 ; \mathrm{p}=0,04)$. Aqueles que tiraram maior nota no questionário teórico no M3 obtiveram também nota maior na atividade prática correspondente a essa etapa (Coeficiente de regressão linear: 1,68; SE: 1,09; $\mathrm{p}=0,001)$.

Após a aula teórica, a maioria dos estudantes (85\%) não apresentou características de nervosismo, irritabilidade, nem estavam bravos frente ao exercício a ser iniciado. Aproximadamente $90 \%$ deles afirmaram estar moderadamente ou muito curiosos em relação à atividade. Cerca de 60\% dos estudantes acreditavam estar moderadamente ou muito preparados para a atividade. Após a atividade prática, o número de estudantes que disse não apresentar nenhum nervosismo caiu discretamente, assim como o grau de curiosidade (gráfico 2). No entanto, a percepção do estudante quanto à confiança de estar bem preparado não se alterou do início para o fim da atividade $(p=0,73)$. Quem acreditava estar bem preparado no M1 teve 3 vezes mais chances de tirar nota acima de 5 no M3 (OD: 3,1; 95\% IC:1,0 - 9,1).

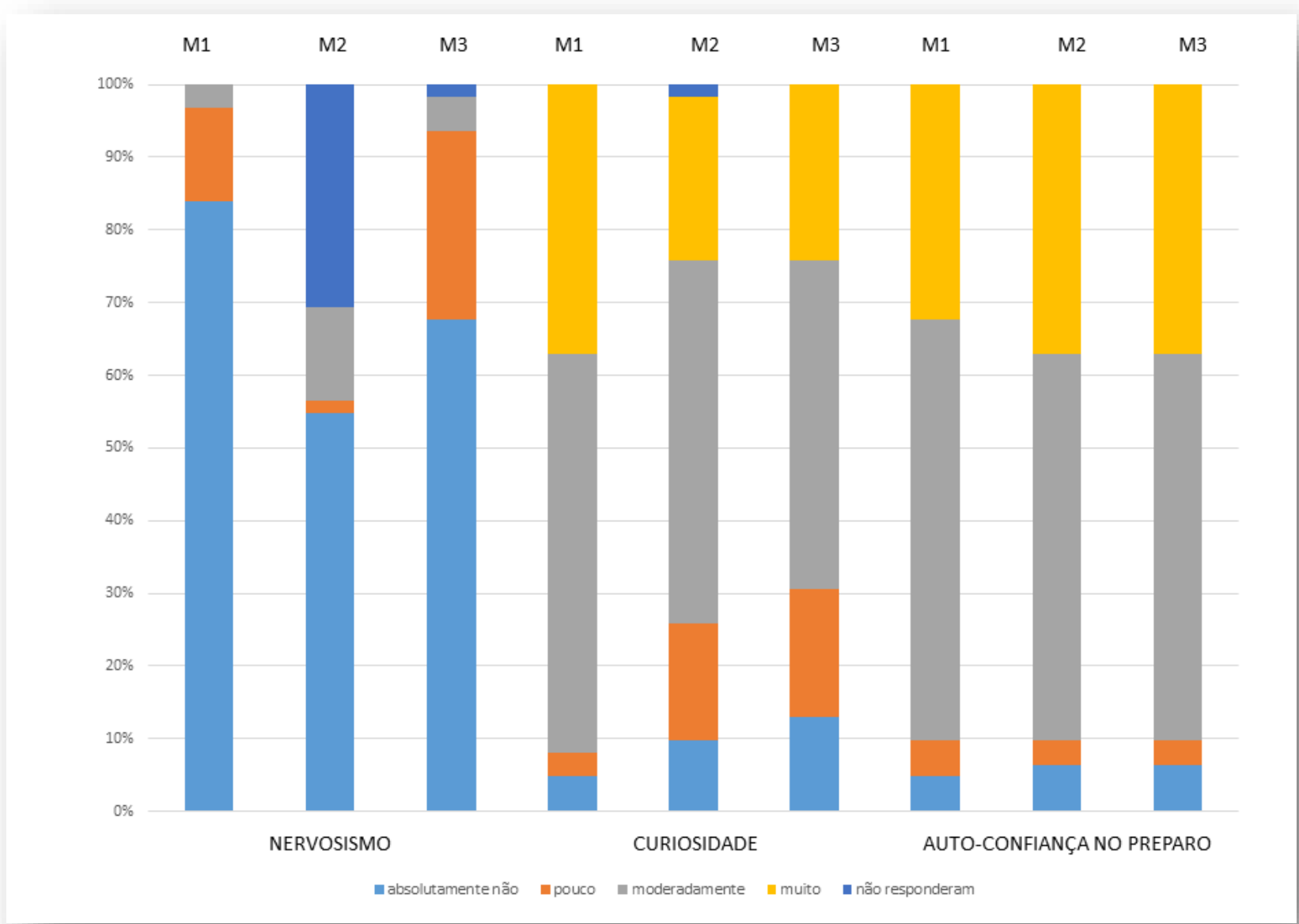

Gráfico 2. Distribuição percentual das respostas dadas pelos estudantes quanto à percepção nos diferentes momentos da atividade. M1: após aula teórica; M2: após avaliação de imagens; e M3: após avaliação de dentes extraídos 


\section{DISCUSSÃO}

As atividades didáticas ministradas sobre o tema "Detecção de lesões de cárie” visam que os estudantes de graduação se tornem aptos a realizar tal prática na clínica e saibam optar pela forma mais correta de tratamento.

O ICDAS é um método que, embora seja indicado para uso no ensino, ainda não é ensinado a estudantes de graduação em todas as instituições, por ser relativamente novo. Por outro lado, é importante utilizar um índice no sentido de melhorar a acurácia e a reprodutibilidade do exame visual das lesões de cárie $^{8}$. Como se sabe, a inclusão de lesões iniciais pode dificultar o exame e induzir maior variabilidade entre os examinadores ${ }^{11,12}$. Nesse sentido, maior é a preocupação quanto ao preparo dos estudantes para uso de tal sistema na prática clínica. Os insights preliminares da atividade prática proposta sugerem que a atividade préclínica, sob diferentes pontos de vista, modificou significativamente o estudante, o que pressupõe que o mesmo tenha ficado mais preparado para utilizar clinicamente esse índice em comparação ao modelo de ensino tradicional.

Quanto ao conhecimento teórico, percebeu-se que o número de respostas corretas aumentou após o treinamento prático com imagens (M2) em relação ao M1. Provavelmente isso se deva à oportunidade de discutir dentro do grupo e com os tutores, esclarecendo possíveis dúvidas que tenham permanecido após a aula teórica. De fato, as atividades práticas têm mostrado influenciar positivamente no conhecimento, habilidades e atitudes entre estudantes das mais diversas áreas ${ }^{13,14}$. Nota-se, no entanto, que não houve melhora no conhecimento teórico na avaliação do M3, em relação ao M2 pois, provavelmente, as principais dúvidas conceituais e de aplicabilidade do sistema tenham sido sanadas no primeiro exercício prático.
A atividade prática-laboratorial possibilitou que o estudante compreendesse melhor aspectos importantes para a classificação das lesões clinicamente, como por exemplo, diferenciação entre os escores 1 e 2, bem como entre escores 3 e 4, o que, especialmente, nesse último caso, levaria a diferenças importantes na tomada de decisões clínicas. No entanto, não se notou melhora no que tange à opinião dos estudantes quanto a possíveis implicações clínicas do erro no diagnóstico. Provavelmente, esse tipo de questão, quando respondida equivocamente, pode requerer que os estudantes tenham maior vivência clínica para identificar e compreender seus erros. Por outro lado, no momento da atividade, os mesmos podem estar muito focados e ávidos por realizar o diagnóstico e não consigam interpretar possíveis situações de erros.

O pior índice de acerto entre os estudantes, a questão 5, revelou a dificuldade em segmentar alguns conceitos assimilados na aula teórica. Quando questionados sobre o que os escores do ICDAS eram capazes de identificar, muitos, mesmo após as atividades práticas, responderam severidade e atividade da lesão de cárie. De fato, a classificação em escores apenas avalia a severidade. Como os estudantes sabem que, na clínica, também é necessário avaliar a atividade da lesão, podem ter escolhido a resposta mais completa, com receio de que os professores pensem que eles não sabem ou que não assimilaram o conhecimento teórico.

Assim, mesmo sendo mais complexo ensinar severidade e atividade em uma mesma atividade prática, talvez seja importante que isso ocorra para auxiliar o estudante na aplicação clínica final desse conhecimento e não confrontar conceitos que se permeiam. Por outro lado, como a atividade de cárie tem um caráter dinâmico, reproduzir integralmente sua avaliação em laboratório é uma tarefa complexa. Por isso, são 
discutidas questões pertinentes à atividade e os estudantes são treinados a identificar características relacionadas ao status diferencial das lesões.

Nas avaliações práticas, com dentes extraídos, houve também melhora no desempenho. Embora a possibilidade de exame de dentes extraídos fosse uma forma de familiarizar os estudantes com o índice antes de fazê-lo clinicamente, o fato de os estudantes terem, em média, errado menos no segundo que no primeiro exame, pode indicar que a atividade laboratorial contribuiu para consolidar conceitos teóricos e sua aplicação. Por outro lado, os estudantes também podem ter melhorado seu desempenho por repetir a atividade e acumular habilidade para a realização dos exames. Portanto, os resultados desse estudo devem ser vistos com parcimônia e estudos com delineamentos mais específicos devem ser conduzidos para avaliar o real impacto dessa proposta educacional para ensino de detecção de cárie na graduação.

O tempo de treinamento (cerca de $2 \mathrm{~h}$ ), bem como o intervalo entre as avaliações foram curtos. Talvez a melhora no desempenho fosse ainda mais expressiva se o tempo de treinamento fosse maior e os estudantes tivessem maior convivência clínica com o uso do índice, ou então, se os estudantes utilizassem algum outro método de fixação de conteúdo em longo prazo. Estudos de seguimento desses estudantes ao final da graduação, poderiam auxiliar na detecção de fatores que permitem sedimentar esse conhecimento em longo prazo ${ }^{15,16}$.

Os estudantes que tiveram maiores notas nas avaliações teóricas do M1 tiveram mais chance de melhor desempenho nas avaliações práticas. Provavelmente este estudante já inicia a atividade mais motivado e seguro, se dedicando mais nas atividades seguintes, refletindo os bons resultados durante as avaliações práticas. Assim, embora a parte prática seja de suma importância, ela não elimina a aula teórica, tanto pela construção de uma bagagem de conhecimento inicial, como pela motivação inicial para o tema $^{17}$.

O melhor desempenho na atividade com imagens em relação à atividade prática final reitera que o estudante empenhado na atividade tende a manter seus bons resultados. A atividade com as imagens assemelhou-se à atividade de $e$ learning $^{9}$, no que diz respeito à classificação das fotografias de lesões cariosas, porém teve o diferencial da interação em grupo, quando os estudantes podiam conversar a respeito de dúvidas, discutir com os colegas e esclarecê-las com os tutores, o que contribui significativamente para a assimilação do conteúdo $^{18}$. No e-learning, muitas vezes o estudante pratica sozinho e sem suporte para tirar dúvidas. Isso também reforça a importância da atividade laboratorial composta por diferentes fases, como proposto. As diferentes etapas tendem a reforçar gradativamente o conhecimento teórico assimilado na aula convencional $^{19}$.

Além de estar apto para utilizar o método, o futuro profissional deve estar seguro de que está bem treinado para classificar clinicamente as lesões de cárie do seu paciente. Os graduandos devem ser capazes de realizar o autojulgamento para compreender no que precisam melhorar e se realmente fixaram os conhecimentos sobre o assunto. No entanto, embora as respostas tenham mostrado que houve melhora tanto no conhecimento teórico como na aplicação prática dos conceitos aprendidos, a percepção do estudante quanto a esse aspecto não mudou entre o início e o fim do estudo.

Apesar de terem conhecimento e estarem aptos a realizar a detecção de lesões de cárie, podem não ser capazes de identificar ou perceber sua melhora do início para o final de todas as 
atividades. Isso sugere que o estudante pode não possuir senso de autocrítica, ou ainda, aquele que já tenha fixado o conteúdo pode também ficar receoso em responder que terá um bom desempenho. Cabe ressaltar que as respostas foram coletadas antes que o gabarito fosse revelado, colaborando para a incerteza sentida ao final da avaliação. Acredita-se, assim, que essa possa ter sido uma razão para a ausência de impacto da atividade na percepção dos estudantes.

Em relação à postura diante da realização da nova atividade, não usual para os mesmos no momento em que se encontravam (final do curso), é notável que no início da atividade a grande maioria estava tranquila e até mesmo curiosa frente ao exercício. O grau de nervosismo aumentou ligeiramente no segundo momento, mostrando que poderiam estar tensos frente à atividade proposta, provavelmente em virtude de temerem o desconhecido. Mesmo tendo sido esclarecidos antecipadamente sobre a atividade, a separação da sala em grupos aleatórios, a expectativa criada frente a uma atividade diferenciada e a insegurança pela interação com os colegas e tutores podem ter gerado uma situação de agitação e também alto grau de curiosidade inicial. Isso, por outro lado, pode refletir interesse por parte dos estudantes frente a uma nova atividade, uma vez que se aproxima da realidade clínica, sendo esse o objetivo de uma metodologia ativa de aprendizagem ${ }^{20}$.

\section{CONCLUSÃO}

A atividade prática-laboratorial contribui para o aprendizado dos estudantes na detecção e avaliação de lesões de cárie, resolvendo também dúvidas conceituais que poderiam existir quando da utilização isolada do método de ensino teórico. No entanto, a percepção dos estudantes sobre seu conhecimento se manteve a mesma em todos os momentos, evidenciando que os mesmos tendem a mostrar posturas positivas em relação à atividade.

\section{AGRADECIMENTOS}

Os autores gostariam de agradecer à FAPESP (2011/18093-0) e ao CNPq (400736/2014-4) pelo apoio financeiro recebido.

\section{ABSTRACT \\ New proposal for teaching/training in caries lesions' detection: insights from the implementation of the method among undergraduate students}

The present study proposes a method for the teaching of the detection and evaluation of caries lesions, using the International Caries Detection and Assessment System (ICDAS) as an auxiliary tool. Sixty-two students in the fourth year of the undergraduate course in Dentistry of the University of São Paulo underwent three training activities for the use of the ICDAS, applied in different moments: regular theoretical class, activity with images projection and practicallaboratory activity. The students answered questionnaires, before and after the activities, to evaluate the knowledge and the perception about them. A practical evaluation was also performed, with evaluation of extracted teeth. After the laboratory exercises, the mean number of correct answers to conceptual questions increased significantly and remained until the end of the laboratory activity. Those who believed to be well prepared at the beginning of the activity had three times more chances to reach a score above 5 in the second moment of the practicallaboratory activity $(\mathrm{OR}=3.1 ; 95 \% \mathrm{CI}=1.0$ 9.1). It was concluded that the practicallaboratory activity contributes to the learning of undergraduate students in the detection of caries lesions, including healing conceptual doubts that may exist after the theoretical class. However, the student's perception may not be impacted by the activity.

Descriptors: Dental Caries. Education, dental. Dentistry

\section{REFERÊNCIAS}

1. Pitts N, Melo P, Martignon S, Ekstrand K, 
Ismail A. Caries risk assessment, diagnosis and synthesis in the context of a European Core Curriculum in Cariology. Eur J Dent Educ. 2011;15(1):23-31.

2. Schulte AG, Pitts NB, Huysmans MCDNJM, Splieth C, Buchalla W. European Core Curriculum in Cariology for undergraduate Dental students. Caries Res. 2011;45(4):336-45.

3. Pitts N. "ICDAS" - an international system for caries detection and assessment being developed to facilitate caries epidemiology, research and appropriate clinical management. Community Dent Health. 2004;21(3):193-8.

4. Ismail A. Visual and visuo-tactile detection of dental caries. J Dent Res. 2004;83:56-66.

5. Burt BA, Kolker JL, Sandretto AM, Yuan Y, Sohn W, Ismail AI. Dietary patterns related to caries in a low-income adult population. Caries Res. 2006;40(6):473-80.

6. Nyvad B, Fejerskov O. Assessing the stage of caries lesion activity on the basis of clinical and microbiological examination. Community Dent Oral Epidemiol. 1997;25(1):69-75.

7. Nyvad B. Diagnosis versus detection of caries. Caries Res. 2004;38(3):192-8.

8. Gimenez T, Piovesan C, Braga MM, Raggio DP, Deery C, Ricketts DN, et al. Visual inspection for caries detection: a systematic review and meta-analysis. J Dent Res. 2015;94(7):895-904.

9. Diniz MB, Lima LM, Santos-Pinto L, Eckert GJ, Zandoná AGF, de Cássia Loiola Cordeiro R. Influence of the ICDAS elearning program for occlusal caries detection on dental students. J Dent Educ. 2010;74(8):862-8.

10. Marteau TM, Bekker H. The development of a six-item short-form of the state scale of the Spielberger State-Trait Anxiety Inventory (STAI). Br J Clin Psychol. 1992;31(3):3016.

11. Assaf AV, de Castro Meneghim M, Zanin L, Tengan C, Pereira AC. Effect of different diagnostic thresholds on dental caries calibration - a 12 month evaluation.
Community Dent Oral Epidemiol. 2006;34(3):213-9.

12. Braga MM, Oliveira LB, Bonini GAVC, Bönecker M, Mendes FM. Feasibility of the International Caries Detection and Assessment System (ICDAS-II) in epidemiological surveys and comparability with standard World Health Organization criteria. Caries Res. 2009;43(4):245-9.

13. Sims MC, Hall DP, Hall N, Archibald AMC, Maxwell SRJ. Teaching medical students prescribing skills: a near-peer approach. Med Educ. 2011;45(11):1144-5.

14. Huntoon KM, McCluney CJ, Wiley EA, Scannell CA, Bruno R, Stull MJ. Selfreported evaluation of competencies and attitudes by physicians-in-training before and after a single day legislative advocacy experience. BMC Med Educ. 2012;12:47. doi: 10.1186/1472-6920-12-47.

15. Bissell V, McKerlie RA, Kinane DF, McHugh S. Teaching periodontal pocket charting to dental students: a comparison of computer assisted learning and traditional tutorials. Br Dent J. 2003;195(6):333-6.

16. Woelber JP, Hilbert TS, Ratka-Krüger P. Can easy-to-use software deliver effective elearning in dental education? A randomised controlled study. Eur J Dent Educ. 2012;16(3):187-92.

17. Katajavuori N, Hakkarainen K, Kuosa T, Airaksinen M, Hirvonen J, Holm Y. Curriculum reform in Finnish pharmacy education. Am J Pharm Educ. 2009;73(8):151.

18. Rashid MS, Sobowale O, Gore D. A nearpeer teaching program designed, developed and delivered exclusively by recent medical graduates for final year medical students sitting the final objective structured clinical examination (OSCE). BMC Med Educ. 2011;11:11. doi:10.1186/1472-6920-11-11.

19. Ashley FA, Gibson B, Daly B, Baker SL, Newton JT. Undergraduate and postgraduate dental students' "reflection on learning": a qualitative study. Eur J Dent Educ. 2006;10(1):10-9.

20. Ruohoniemi M, Parpala A, Lindblom-Ylanne 
S, Katajavuori N. Relationships between students' approaches to learning, perceptions of the teaching-learning environment, and study success: a case study of third-year veterinary students. J Vet Med Educ. 2010;37(3):282-8.

\author{
Mariana Minatel Braga \\ e-mail:mmbraga@usp.br \\ Departamento de Ortodontia e Odontopediatria \\ da USP/SP \\ Av. Prof. Lineu Prestes, 2227 Cidade Universitária \\ 05508-000 São Paulo/SP
}

Correspondência para: 